\title{
INVESTIGACIONES
}

\section{Neurociencias, emociones y educación superior: una revisión descriptiva*}

\author{
Neurosciences, Emotions and Higher Education: \\ A Descriptive Review
}

\author{
Hernando Barrios Tao ${ }^{a}$, Carolina Gutiérrez de Piñeres Botero ${ }^{a}$ \\ ${ }^{a}$ Facultad de Educación y Humanidades, Universidad Militar Nueva Granada, Colombia. \\ hernando.barrios@unimilitar.edu.co,carolinagdep@yahoo.com
}

\section{RESUMEN}

El desarrollo de habilidades socioemocionales desafía los escenarios educativos, laborales y sociales. Los avances investigativos de las neurociencias evidencian el vínculo entre cognición y emoción, y aportan fundamentos para comprender las bases neuronales y psicobiológicas de los procesos educativos. El objetivo del artículo de revisión es presentar hallazgos relevantes en el campo de las neurociencias emocionales y sociales, que podrían contribuir al diseño e implementación de prácticas de enseñanza-aprendizaje basadas en la evidencia, el desempeño académico, el desarrollo curricular, las políticas de educación, así como al fortalecimiento del desarrollo de habilidades sociales, emocionales y cognoscitivas. Los resultados indican la necesidad de incluir las emociones como parte fundamental en la formación integral y ofrecen bases para comprender el desarrollo y las limitaciones de los aspectos neuronales en los procesos educativos. La evidencia empírica, desde las neurociencias, es aún incipiente y los desarrollos investigativos permanecen aún en los laboratorios.

Palabras claves: educación, enseñanza-aprendizaje, competencias emocionales, habilidades socioafectivas.

\begin{abstract}
The development of socio-emotional skills challenges the educational, work and social scenarios. The research advances of the neurosciences show the link between cognition and emotion and provide foundations to understand the neuronal and psychobiological bases of educational processes. The aim of the review article is to present relevant findings in the field of emotional and social neurosciences, which could contribute to the design and implementation of teaching-learning practices based on evidence, academic performance, curricular development, education policies, as well as the strengthening of the development of social, emotional and cognitive skills. The results indicate the need to include emotions as a fundamental part of integral education and offer bases to understand the development and limitations of neuronal aspects in educational processes. The empirical evidence, from the neurosciences, is still incipient and the research developments still remain in the laboratories.
\end{abstract}

Key words: education, teaching-learning, emotional competences, socio-affective skills.

\footnotetext{
* El artículo se asocia con el Proyecto de Investigación con Código INV-HUM-2606: "Neurociencias, educación y emociones. Fase 1: Revisión narrativa", financiado por la Vicerrectoría de Investigaciones de la Universidad Militar Nueva Granada, Bogotá - Colombia.
} 


\section{INTRODUCCIÓN}

La universidad es un escenario que supera la transmisión de saberes, la capacitación de científicos expertos en el manejo de tecnologías y se orienta a formar profesionales con habilidades para innovar, investigar y ser agentes de mejoramiento y cambio social, a través de procesos de formación que involucren todas las facetas del ser humano (Ariza y Ocampo, 2005). En el cumplimiento de su misión y en el marco de una formación integral, la universidad debe implementar diversas estrategias, que se ajusten a las constantes transformaciones sociales, políticas, económicas y culturales de su contexto. Estas estrategias se orientan a los siguientes objetivos: a) facilitar la adaptación y permanencia de los estudiantes a la vida universitaria, especialmente durante los primeros semestres de carrera; b) generar alternativas para el desarrollo de las competencias generales y específicas que deben adquirir los futuros profesionales. Ambos objetivos requieren de planeación e implementación de acciones, planes y programas (Cerezo, Ceca y Blanco, 2018), que garantizan una educación superior con calidad y excelencia, para lo cual es esencial el compromiso y el papel que desempeñan cada uno de los actores que integran las instituciones de educación superior.

La formación integral integra habilidades personales y sociales que fortalecen las dimensiones del ser, comunicar y convivir, claves en el desarrollo armónico y en el desempeño social de los actores educativos. De manera particular, las denominadas habilidades emocionales adquieren relevancia más allá de los procesos educativos y se potencia su fortalecimiento en los ámbitos empresariales, orientado al desempeño laboral y al aporte con el desarrollo sostenible. Tanto la Organización para la cooperación y el desarrollo económico (OECD) como el Foro económico mundial (WEF) destacan escenarios, informes y comunicados sobre experiencias de fortalecimiento de estas habilidades y la necesidad de políticas para su desarrollo e implementación. El Informe WEF (2016) así como el Foro 2018, refirieron el necesario desarrollo de habilidades y actitudes como pensamiento crítico, creatividad, cooperación, adaptabilidad, conciencia social y cultural, entre otras, en los procesos de aprendizaje socioemocional. Estabilidad emocional, conciencia, apertura experiencial, afabilidad, extraversión, son cinco dimensiones consideradas en el marco conceptual del Documento No 173 de la OECD (Chernyshenko, Kankaraš y Drasgow, 2018).

El contexto latinoamericano ha desplegado esfuerzos para el desarrollo de diversas competencias como el caso de la adopción e implementación del Proyecto Tuning, concebido como una estrategia pedagógica centrada en el estudiante y en el reconocimiento de su protagonismo en su propia capacidad de aprender, así como en sus competencias emocionales para relacionarse con otros: habilidades interpersonales, capacidad de trabajo en equipo y ante nuevas situaciones, entre otras (Beneitone et al., 2007). A través de esta pedagogía es posible diseñar un currículo y estructurar un plan de estudios más flexible (Gutiérrez, Zalba y Deaminic, 2010), cuyos resultados se traducen en comportamientos observables durante el ejercicio de una profesión, en la contribución al éxito en el desarrollo profesional (Lévy-Leboyer, 2000) y en el ejercicio de aplicación de conocimientos en circunstancias críticas (Gallart, 1997). Asimismo, sus frutos se perciben en la visión de una persona profesional capacitada para actuar y contribuir a la solución de problemas de forma adecuada con su contexto, debido a que moviliza recursos cognoscitivos, emocionales y sociales que considera pertinentes (Le Boterf, 2001) y se adapta a las constantes y 
cambiantes demandas de los entornos laborales (Arroyo, 2012; Beneitone et al., 2007; Cerezo et al., 2018), de forma eficaz y eficiente (Lasnier, 2000).

Esta estrategia pedagógica traza un horizonte en la transformación de prácticas tradicionales de enseñanza-aprendizaje, mediante la inclusión de procesos que en algunos escenarios aún son considerados poco relevantes: emociones y cognición social. El objetivo del artículo de revisión descriptiva es presentar algunos de los principales hallazgos en el campo de las neurociencias emocionales y sociales, que podrían ser relevantes en la educación superior, tanto para el diseño e implementación de prácticas de enseñanzaaprendizaje basadas en la evidencia, de estrategias que faciliten la adaptación a la vida universitaria, el desempeño académico y el desarrollo de currículos, así como para el fortalecimiento y desarrollo de competencias sociales, emocionales y cognoscitivas que favorezcan un mejor desempeño profesional y una orientación para las políticas de educación superior (Carew y Magsamen, 2010).

\section{ASPECTOS TEÓRICOS: NEUROCIENCIAS, EMOCIONES Y COGNICIÓN}

Los avances en el campo de las neurociencias evidenciaron el vínculo indiscutible entre emociones y cognición, emociones y aprendizaje (Immordino-Yang, 2011; Meltzoff et al., 2009), emociones y decisiones morales (Immordino-Yang y Damasio, 2007; Han et al., 2016; Pletti et al., 2017). Asimismo, se ha mostrado que las emociones desempeñan un rol preponderante sobre el pensamiento racional y la toma de decisiones (Fuster, 2003; Prada y González, 2014; Torres et al., 2013; Vohs et al., 2008; George y Dane, 2016).

La hegemonía sostenida, hasta no más de 30 años, por la neurociencia cognitiva sobre el estudio de los procesos de enseñanza, aprendizaje y formación de competencias, impulsó el desarrollo de investigaciones a través de las cuales se pretendía comprender y explicar funciones como la memoria, la toma de decisiones, el lenguaje y la atención (Gazzaniga, 2004), entre otras razones porque se consideraba que la forma de medir el aprendizaje era a través del comportamiento (Wolfe, 2006). Este hecho demoró el abordaje neurocientífico de las emociones, el comportamiento y la cognición social, ya que se suponía que estos procesos poco o nada influían en el aprendizaje o en la formación de competencias.

Estudios de laboratorio con animales (LeDoux, 2012), investigaciones en pacientes con daño cerebral (Damasio, 1994), investigaciones sobre el impacto de la soledad y el aislamiento sobre el comportamiento social y la salud mental (Uchino, Cacioppo y Kiecolt-Glaser, 1996; Decety y Ickes, 2009; Cacioppo y Cacioppo, 2013; Cacioppo et al., 2009), estudios en pacientes con cerebro dividido (LeDoux, Wilson y Gazzaniga, 1978) y experimentos llevados a cabo sobre las emociones y el comportamiento social (Zajonc, 1980), han permitido reconocer que las emociones y el comportamiento social, podrían tener un papel más relevante sobre la cognición, el funcionamiento social, el bienestar psicológico, la calidad de vida, el aprendizaje y la toma de decisiones, en la formación en contextos de educación preescolar, primaria y secundaria (Immordino-Yang y Damasio, 2007; Rychen y Salganik, 2006; Bar-On y Parker, 2000; Taylor et al., 2017), de lo que hasta entonces se había pensado.

Del mismo modo, diversos estudios sugieren que las emociones son indispensables para el desarrollo de habilidades y competencias no solo cognitivas sino también sociales, ya que aquellas guían los juicios y las acciones (Damasio, 2005). Asimismo, se 
ha evidenciado que las dimensiones sociales y las emociones, son moduladoras de los procesos de enseñanza aprendizaje (Vuilleumier, 2005), y que toda actividad humana y animal se podía explicar a partir del estudio del sistema nervioso.

Los anteriores hallazgos se condujeron desde las neurociencias afectivas y emocionales (Panksepp y Biven, 2012) y las neurociencias sociales (Cacioppo y Cacioppo, 2013), cuyo desarrollo se ha visto favorecido por el avance en los dispositivos para estudiar el cerebro en acción y por los avances en metodologías como las técnicas de neuroimagen, los estudios de laboratorio con animales no humanos, los test y las baterías neuropsicológicas. El estudio con personas con condiciones neuropsiquiátricas y neurológicas también han posibilitado una aproximación a las bases neuronales que subyacen en los procesos afectivos, emocionales y sociales (Ibanez et al., 2014).

Es importante resaltar que cuando el desarrollo emocional adquieren un papel relevante dentro de los procesos de enseñanza-aprendizaje a los estudiantes se les facilita tomar decisiones libres, responsables y autónomas, teniendo en cuenta las consecuencias de sus decisiones sobre la vida de otros (dimensión ética); es capaz de trascender más allá de su existencia para entrar en contacto con la existencia de los demás (dimensión espiritual); es capaz de comprender a aplicar creativamente los conocimientos haciendo reflexiones críticas e innovadores (dimensión cognoscitiva); es capaz de relacionarse positivamente consigo mismo y con los demás, a partir de la gestión emocional (dimensión afectiva); es capaz de usar el lenguaje verbal y no verbal para trasmitir y comprender mensajes con sentido y significado (dimensión comunicativa); es capaz de expresar creativamente su sensibilidad y apreciar y transformar el entono (dimensión estética); y es capaz de asumir un compromiso solidario en la construcción de una sociedad más justa (dimensión sociopolítica) (Paoloni, 2014).

\section{METODOLOGÍA}

Se realizó una revisión descriptiva en la búsqueda de avances en las relaciones entre emociones, neurociencias y educación superior. La búsqueda de artículos se realizó en bases y sistemas internacionales, Psyarticles, Ebscohost, ScienceDirect, Psicodoc y Proquest, mediante el uso de las siguientes palabras y expresiones: "neuroeducación", “emociones”, “emociones y cognición”, “estrategias pedagógicas”, "psicopedagogía de las emociones", "neurofisiología de las emociones", "pedagogía y emociones", "neuroscience and emotions", "soft skill and affective nueorscience", "brain based teaching Strategies", "effects of educational interventions through brain imaging".

Como criterios de inclusión se tuvieron en cuenta artículos publicados entre 20082018, que hubieran sido en población de estudiantes universitarios de pregrado; los diseños investigativos debían ser revisiones sistemáticas o estudios experimentales; los instrumentos podían incluir el uso de EEG, evaluaciones neuropsicológicas, eye-tracking, FIMR, RM o Electromiografía. En cuanto al estado de las publicaciones, debían ser estudios publicados en revistas indexadas. Con relación al reporte de resultados se seleccionaron estimaciones individuales del efecto para estudios primarios y estimaciones combinadas para meta-análisis; estudios publicados en inglés o español. Los criterios de exclusión fueron: población de estudiantes de educación básica primaria y secundaria, revisiones selectivas, reseñas y artículos de reflexión. 


\section{RESULTADOS}

De acuerdo con los criterios de inclusión y exclusión, se revisaron 150 publicaciones de las que se seleccionaron 70. Los resultados de la revisión descriptiva se presentan a continuación en tres apartados con su respectiva discusión. Un primer bloque reúne líneas teóricas sobre las relaciones entre cognición y emoción. El segundo, las estructuras cerebrales y los neurotransmisores involucrados en la diada cognición-emoción. En el tercer bloque se describen líneas investigativas en neurociencias aplicadas a la educación y enseñanza-aprendizaje.

\subsection{LÍNEAS TEÓRICAS SOBRE RELACIONES ENTRE EMOCIÓN Y COGNICIÓN}

No existe consenso sobre la conceptualización de las emociones, pero se acepta que incluyen diversos aspectos: motor-expresivo, sensorial-perceptual, autonómico-hormonal, cognitivoatencional y afectivo-sentimental (Panksepp, 2003). Las emociones se pueden entender como sentimientos subjetivos asociados al placer o al dolor (estados mentales), como expresiones de respuestas somáticas y autónomas asociados a estados de activación fisiológica (estados biológicos), y como respuestas de supervivencia (estados biológicos funcionales) que generan tres tipos de comportamientos: ataque, huida o conductas de congelamiento. En todo caso, son concebidas como experiencias multidimensionales que involucran al menos tres canales de respuesta: cognitivo, conductual y fisiológico, cada uno de los cuales puede adquirir una primacía diferente, según el estímulo que la originó. Estas experiencias originan distintos aspectos de la emoción: sus experiencias conscientes (sentimientos), sus comportamientos causados (expresiones emocionales) y la capacidad humana para atribuir y reconocerlas en miembros de la misma especie o de otras especies (Adolphs, 2017).

Las emociones pueden dividirse al menos en dos categorías: emociones primarias y secundarias. Las primarias, también conocidas como emociones básicas, son disposiciones básicas de acción determinadas por mecanismos biológicos innatos, lo que determinaría su universalidad y su presencia tanto en los seres humanos como en otros animales no humanos. Estas emociones se producen y procesan de forma automática como respuesta a estímulos ambientales específicos y su presencia en los seres garantiza su supervivencia y adaptación. Las emociones primarias, ira, tristeza, alegría, sorpresa y miedo, se vinculan con funciones vegetativas específicas (latido del corazón, presión sanguínea, ritmo cardíaco, etc.) y preparan las personas para responder a las demandas del entorno (Damasio, 1994; LeDoux, 1996). Damasio et al. (1996) propusieron a partir de esta noción la hipótesis del marcador somático. De acuerdo con esta hipótesis, existen ciertas señales que producen marcas a partir de procesos biorreguladores, e influyen en los procesos de respuesta a los estímulos, de forma abierta o consciente o de forma automática no consciente.

Las emociones secundarias están integradas por dos o más emociones básicas y se originan en las relaciones sociales, lo que determina su variación de una cultura a otra. Estas emociones incluyen la compasión, la envidia, el orgullo, los celos, la culpa, la vergüenza, la gratitud, entre otras. Jankowski y Takahashi (2014) las definen como un conjunto de estados afectivos que se producen durante las interacciones sociales e integrales para promover conductas socialmente apropiadas y desalentar las socialmente inapropiadas.

Dentro de las emociones sociales, se ubican aquellas determinantes de la conducta moral, lo que originó el concepto de emociones morales (Moll, De Oliveira-Souza y 
Eslinger, 2003) caracterizadas por su relación con los intereses de los individuos o de la sociedad, su rol en la promoción de comportamientos socialmente aceptables y la inhibición de comportamientos inaceptables. Estas emociones morales son el producto del conocimiento social contextual e incluyen, además de las emociones vinculadas con la dimensión moral, las denominadas Emociones Autoconscientes (SCE-Self-Conscious Emotions), asociadas con la obediencia o la transgresión de las normas sociales, así como también las denominadas emociones de la fortuna (FOE), evocadas a través de la comparación social.

El rol otorgado a cada una de las dimensiones de las emociones, con relación a los procesos cognitivos, origina diferentes modelos comprensivos sobre las emociones (Chóliz, 2005). Al menos tres escuelas neurocientíficas han emergido a partir de los diferentes modelos comprensivos, basados en los procesos neuronales. Estos modelos se han interesado por comprender y explicar las relaciones entre procesos cognitivos y procesos emocionales, a partir de bases neuronales y psicobiológicas. La neurociencia conductual niega que las capacidades afectivas existan como aspectos inherentes a la vida de humanos y animales, y argumenta que, en el caso de suponer que las emociones existen, serían eventos secundarios que no tienen la capacidad de influir sobre los procesos cognitivos (LeDoux, 1996; Gray, 1990; Rolls, 2000).

Por su parte, la neurociencia cognitiva concibe los afectos como una parte inherente de las funciones cognoscitivas humanas (Damasio, 1999). Finalmente, las neurociencias afectivas proponen: a) que los afectos se originan en estructuras subcorticales; b) que las emociones median la generación de los sentimientos; c) que existe una diferencia entre los procesos cognitivos y los procesos emociones desde el punto de vista neuroanatómico, neurofuncional, neuroquímico y neurobiológico; y d) que los procesos emocionales le ayudan al organismo a tomar decisiones (Panksepp, 2003). Estas tres escuelas originaron diversas teorías que intentan explicar la relación entre emociones y cognición.

Estas teorías deberían ser consideradas al momento de intentar desarrollar comprensiones del comportamiento, la cognición, la emoción y la naturaleza del aprendizaje humano, y deberían presentarse de manera explícita para demarcar epistemológica y teóricamente las implicaciones que tiene determinada investigación para el desarrollo de nuevos modelos de aprendizaje, de los métodos de enseñanza, de la creación de principios de diseño para el desarrollo de materiales de instrucción, entre otros.

Algunas de estas teorías permiten una mayor comprensión sobre la interrelación entre cognición, emoción e interacción social, razón por la cual algunas teorías incorporan dos nuevas variables: la interacción social y las emociones como mediadoras de estas relaciones (Howard-Jones, 2011). Los supuestos teóricos consideran uno o más componentes, lo que ha derivado en una clasificación de las diferentes teorías: cognitivas, social-constructivistas, biológicas e integradoras.

Uno de los primeros autores que trató de entender y explicar las emociones fue Charles Darwin, quien en 1872 publicó La expresión de las emociones en hombres y animales. Darwin sugirió que existían distintas clases de expresiones emocionales, tanto a nivel somático como visceral, que se conservaban más o menos estables a nivel filogenético y que evolucionaron según su función adaptativa.

Posteriormente, una de las primeras teorías propuestas para explicar la relación entre cognición y emoción, fue la Teoría de las bases fisiológicas de la emoción de James y Lange. Esta teoría postula que la experiencia emocional se basa en la conciencia de las respuestas 
fisiológicas (tensión muscular, aumento de la frecuencia cardíaca, llanto, sudoración). De acuerdo con James (1884), las emociones son la percepción de los cambios corporales que surgen automáticamente ante la presencia de un estímulo específico, razón por la cual, por ejemplo, las personas podían saber que estaban tristes porque lloraban.

Hacia 1927, Cannon y Bard, fisiólogos estadounidenses, propusieron su teoría en la que controvertían la teoría propuesta por James y Lang. De acuerdo con estos autores, las emociones son un productor cognitivo. Contrario a la teoría de James y Lang, Cannon y Bard conceden mayor relevancia al Sistema Nervioso Central y al rol de las estructuras corticales en la regulación de la respuesta emocional (Deak, 2011).

En 1937, Papez argumentó que el centro de las emociones no estaba suscrito a un solo sistema, sino que estaba formado por un circuito que involucraba estructuras corticales y subcorticales: tálamo, hipotálamo, cuerpos mamilares, giro cingular, amígdala, septum, bulbo olfativo e hipocampo. Asimismo, explicó que las aferencias sensoriales de este circuito originaban tres rutas: la primera dirigida a la corteza cerebral, relacionada con las cogniciones responsables de regular las respuestas emocionales; la segunda dirigida hacia los ganglios basales se relacionaba con los movimientos implicados en una respuesta emocional; y la tercera dirigida hacia el hipotálamo era responsable de las sensaciones (LeDoux \& Phelps, 2000).

En la década del 50', Lindsley (1951) propuso la Teoría de la activación de las emociones y sostuvo que estas son un complejo fenómeno comportamental que involucra diversos niveles de integración química y neuronal que pueden ser medidos mediante la actividad electro-encefalográfica. Este psicólogo y fisiólogo, pionero en el estudio funcional del cerebro, descubrió el Sistema de Activación Reticular. Explicó que el Sistema Activador Reticular Ascendente (SARA) era responsable de la homeostasis de activación. Asimismo, postuló la existencia de un continuo de activación entre la no emoción y la emoción intensa, que lleva a las personas a estar en estados de aletargamiento o sueño y de varios rangos de comportamiento motivado.

En 1960, la psicóloga post-behaviorista Magda Arnold, pionera de la teoría moderna de la cognición emocional, con su Teoría de la valoración o de la evaluación cognitiva sugirió que la activación de una respuesta emocional se vincula con la valoración que se hacía de los estímulos presentes en el entorno y que conducían a las personas a llevar a cabo conductas de acercamiento o distanciamiento. En esta misma década, Schachter y Singer (1962) plantearon la Teoría de los dos factores, en la cual señalan que un estado emocional es el resultado de una activación fisiológica y de una etiqueta cognitiva asociada con dicha activación. Asimismo, sostuvieron que la emoción depende de una doble apreciación: como se evalúa el suceso y como se identifica la respuesta fisiológica. Desde esta postura, la cognición ejerce una función de dirección y de interpretación de la emoción, teniendo como referente la experiencia pasada. En este caso, la interpretación personal de las respuestas fisiológicas sería el factor más relevante en la emoción, más que la información orgánica.

La Teoría de las emociones diferenciales (DET) (Izard, 1971; 1979) considera que las emociones se expresan a través de tres canales: neuronal, expresivo y experiencial. Izard (1971), estudió ocho emociones básicas (enojo, alegría, sorpresa, miedo, angustia, disgusto, interés y vergüenza) en diferentes culturas y observó que la capacidad para interpretarlas e identificarlas era innata y universal, y se complejizaban más a lo largo de los primeros años de la vida. Izard considera que las emociones son resultado de la 
evolución y del desarrollo neurobiológico, cumpliendo un papel relevante en la adaptación. En esta misma línea, Plutchik (1980) propone la Teoría psicoevolutiva, según la que las emociones son reacciones del organismo para permitir una mejor adaptación. A un grupo de emociones denominó primarias (desagrado, ira, anticipación, alegría, aceptación, miedo, sorpresa y tristeza), y a otro conjunto de emociones, derivadas de la interacción de dos o más emociones primarias, denominó secundarias. De la unión entre tristeza y desagrado surgiría el remordimiento; entre desagrado e ira, el desprecio; entre ira y anticipación, la agresividad; entre anticipación y alegría, el optimismo; entre alegría y aceptación, el amor; entre aceptación y miedo, la sumisión; entre miedo y sorpresa, el terror; y entre sorpresa y tristeza, la decepción. Ambos conjuntos de emociones tendrían una valencia positiva o negativa. Emociones como amor, compasión y sentimientos de pertenencia, generan aceptación y brindan a las personas la sensación de inclusión, por el contrario, emociones negativas como hostilidad e ira, conducen a la aversión y el rechazo.

Tanto la Teoría de la primacía afectiva de Zajonc (1980), como Le Doux (1989) conciben las emociones como independientes de los procesos cognitivos, se procesan a través de vías subcorticales y anteceden los procesos cognitivos. Paralelamente, Lazarus (1982) propone que la cognición es precondición necesaria para el procesamiento de emociones. Las experiencias emocionales son resultado de una transacción entre persona y ambiente, en la que el sujeto evalúa qué tanto puede estar implicado su bienestar ante una situación presentada.

En la década del 90', MacLean (1990) propuso la Teoría del cerebro triuno con el fin de explicar el funcionamiento del cerebro en términos evolutivos y de brindar una explicación a lo que se denominó el instinto de supervivencia. El cerebro evolucionaría a partir de tres capas: 1) Cerebro reptiliano: capa más antigua, responsable de las respuestas innatas automáticas y acciones mecánicas relacionadas con la supervivencia: respiración, sueño, reproducción y búsqueda de alimento; 2) Cerebro mamífero antiguo o sistema límbico: responsable de la conservación de la especie e incluiría estructuras neurales implicadas en las emociones básicas y en las respuestas de lucha o huida; 3) Cerebro mamífero nuevo o neocórtex: sería la más evolucionada y responsable de las estrategias racionales y de la capacidad verbal.

Unos años después, Lang (1995) formula el Modelo bioinformacional de las emociones, según el cual las emociones son disposiciones para la acción, cuyo origen es un estímulo que resulta significativo para el individuo. A partir de la activación del sistema apetitivo o del sistema defensivo, la emoción prepara al organismo para emitir un comportamiento de aproximación o de escape. Las respuestas generadas por las emociones serían de tres tipos (cognitivas, motoras y neurofisiológicas) y vinculadas con tres dimensiones básicas de la estructura afectiva: valencia afectiva (desde lo agradable hasta lo desagradable); activación o arousal (desde la excitación hasta la calma); y dominación o control (grado de control percibido que puede tener sobre su respuesta emocional).

Finalmente, la propuesta más reciente es la de Damasio (1999) quien postula la Hipótesis del marcador somático, integrando procesos cognitivos, emocionales y sistemas neuroanatómicos, para explicar el vínculo entre el procesamiento de emociones y la capacidad para decidir en función de las potenciales consecuencias futuras de la conducta. De acuerdo con esta teoría, los sentimientos surgen cuando ciertas zonas del cuerpo reciben una realimentación sobre la excitación emocional. La emoción actúa como un marcador somático, producido a partir de procesos de homeostasis y autorregulación, lo que produce 
como consecuencia un cambio fisiológico tanto en el cuerpo como en regiones cerebrales implicadas en la representación de los estados emocionales. Un marcador somático se produce a partir de uno de dos tipos de eventos: inductores primarios e inductores secundarios. Los primeros son un conjunto de estímulos que, a través de mecanismos innatos o aprendidos, se han asociado a estados de placer o aversión, de manera que cuando uno de estos estímulos o algunos que tenga características similares al que dio origen a la marca somática inicial, esté presente en el entorno, se producirá una respuesta emocional automática. En este caso la amígdala opera como disparador de las señales emocionales. Los inductores secundarios son respuestas emocionales generadas a partir de la presentación de un estímulo que evoque recuerdos personales o hipotéticos asociado al placer o a la aversión que produjo un inductor primario. En ambos casos se forjan patrones de conducta y guías de acción que orientan procesos de toma de decisiones.

\subsection{ESTRUCTURAS CEREBRALES Y NEUROTRANSMISORES INVOLUCRADOS EN LA DIADA COGNICIÓN-EMOCIÓN}

Gracias a los avances en neurociencias y a las tecnologías para estudiar el cerebro en acción, hoy es posible conocer las bases neuroestructurales y neurofuncionales de la diada cognición-emoción. Los resultados de un número significativo de investigaciones han permitido identificar las estructuras cerebrales y los neurotransmisores que participan en los procesos en los que se articulan cognición y emoción y que interactúan a través de circuitos neuronales integrados por estructuras ubicadas en regiones cerebrales corticales y subcorticales, organizados jerárquicamente, que permiten la comunicación selectiva entre las neuronas y proporcionan un flujo preciso de información. En este sentido, Pessoa (2017) propone el concepto de "sistemas funcionalmente integrados" que harían parte de una red de interacciones a gran escala, que involucra sustratos anatómicos y funcionales de sistemas cortical-subcorticales a partir de los cuales se entiende la organización de las emociones en el cerebro. Esta red está conformada por un sistema integrado de cortezaamígdala, que se cruza con los sistemas que involucran el estriado ventral / accumbens, el tabique, el hipocampo, el hipotálamo, el tronco encefálico, el tálamo, la ínsula y la corteza cingulada. Estos componentes, regulados por un conjunto de neurotransmisores, organizan la expresión de la experiencia emocional a partir de varios sistemas. Son sistemas descendentes que controla los efectores motores somáticos y viscerales, controlando los movimientos voluntarios, las expresiones emocionales, entre otros. En las Tablas 1 y 2 se presentan algunas de las funciones que cumplen estas estructuras y los neurotransmisores vinculados con este circuito. 
Tabla 1. Principales estructuras cerebrales y sus funciones relacionadas con las emociones

\begin{tabular}{|c|c|}
\hline Estructura & Función \\
\hline $\begin{array}{l}\text { Corteza Cingulada } \\
\text { Anterior (CCA) }\end{array}$ & $\begin{array}{l}\text { - Relacionada con la inhibición de la respuesta emocional } \\
\text { - Asociada con el mejor reconocimiento de las expresiones faciales } \\
\text { de enojo }\end{array}$ \\
\hline $\begin{array}{l}\text { Hemisferio Cerebeloso } \\
\text { Derecho }\end{array}$ & $\begin{array}{l}\text { - Parece estar asociado con una experiencia placentera en respuesta } \\
\text { a estímulos evocadores de la felicidad } \\
\text { Desempeña un papel en el procesamiento sensorial y regulación } \\
\text { de las emociones, regulación de estado de ánimo; una lesión en } \\
\text { esta región puede dar origen a el síndrome cerebeloso cognitivo- } \\
\text { afectivo } \\
\text { - Relacionado de forma bidireccional con regiones que favorecen la } \\
\text { percepción del material socialmente relevante }\end{array}$ \\
\hline Complejo Amigdaliano & $\begin{array}{l}\text { - Relacionado principalmente con las respuestas al miedo y } \\
\text { vinculada con la experiencia subjetiva de peligro, temor inminentes } \\
\text { o ira. Se activa con mayor fuerza por expresiones faciales de } \\
\text { miedo que por otras expresiones faciales, incluida la ira } \\
\text { - Un daño en esta región puede afectar el reconocimiento emocional } \\
\text { a través de las expresiones faciales del miedo } \\
\text { - Función moduladora de almacenamiento de información que tiene } \\
\text { lugar en otras estructuras, especialmente relacionadas con el } \\
\text { miedo } \\
\text { - En esta región se presentan mecanismos de plasticidad sináptica } \\
\text { - Involucrada en la asociación de estímulos con castigos y } \\
\text { recompensas primario, así como con la generalización del afecto } \\
\text { negativo por ejemplo ante imágenes de animales desagradables. } \\
\text { - Se activa en respuesta a señales que conllevan amenaza }\end{array}$ \\
\hline $\begin{array}{l}\text { Ínsula, corteza } \\
\text { cingulada o Isla de Reil }\end{array}$ & $\begin{array}{l}\text { - La ínsula tiene conexiones bidireccionales con los lóbulos frontal, } \\
\text { parietal y temporal; la circunvolución cingulada y estructuras } \\
\text { subcorticales tales como la amígdala, tronco encefálico, tálamo y } \\
\text { ganglios basales } \\
\text { Estas conexiones sirven para la integración de funciones } \\
\text { autonómicas, víscerosensoriales, vísceromotoras y límbicas } \\
\text { - Se divide en una porción anterior agranular (ínsula anterior, AIC), } \\
\text { una porción disgranular media (insula media), y una porción } \\
\text { granular posterior (ínsula posterior), cada una de las cuales se } \\
\text { conecta de forma única con otras regiones cerebrales y así mismo } \\
\text { tiene unas características funcionales particulares } \\
\text { - La ínsula posterior está involucrada en funciones somato-motoras } \\
\text { La corteza insular anterior (AIC) se relaciona con información } \\
\text { autonómica e interoceptiva. También está involucrada en la } \\
\text { empatía, la compasión, la equidad y la cooperación, razón por la } \\
\text { cual se cree que desempeña un importante papel en las emociones } \\
\text { sociales y de en las decisiones que involucran juicios morales } \\
\text { La ínsula cumple una función crítica en la conciencia emocional }\end{array}$ \\
\hline
\end{tabular}




\begin{tabular}{|l|l|}
\hline Corteza Orbitofrontal & $\begin{array}{l}\text { Asociada con el mejor reconocimiento de las expresiones faciales de } \\
\text { enojo, lo que probablemente explica su papel en las respuestas } \\
\text { reguladoras automáticas que controla la intensidad de la ira expresada. }\end{array}$ \\
\hline $\begin{array}{l}\text { Corteza Prefrontal } \\
\text { Ventromedial } \\
\text { (VMPFC) }\end{array}$ & $\begin{array}{l}\text { Involucrada en la regulación del comportamiento social y moral } \\
\text { (inadecuación social, falta de tacto social, violaciones morales } \\
\text { graves) }\end{array}$ \\
$\begin{array}{l}\text { Vinculada con la organización del comportamiento moral } \\
\text { (predicción de resultados, aprendizaje asociativo y evaluación } \\
\text { flexible de contingencias conductuales) }\end{array}$ \\
\hline
\end{tabular}

Fuente. Elaboración propia.

Tabla 2. Principales neurotransmisores y sus funciones relacionadas con las emociones

\begin{tabular}{|c|c|}
\hline Neurotransmisores & Función \\
\hline Dopamina & $\begin{array}{l}\text { - Neuromodulador de las regiones neuronales involucradas en el } \\
\text { aprendizaje emocional, la percepción y la formación de la memoria } \\
\text { - Involucrada en la codificación y la expresión del aprendizaje } \\
\text { emocionalmente sobresaliente y la formación de la memoria } \\
\text { - Capacidad para procesar con precisión la información sensorial } \\
\text { emocionalmente relevante } \\
\text { - Asociada con emociones placenteras } \\
\text { - Participa en la regulación del estado de ánimo positivo }\end{array}$ \\
\hline Serotonina & $\begin{array}{l}\text { - Juegan un papel importante en el aprendizaje y la memoria } \\
\text { Está vinculada con los aspectos emocionales, motivacionales y } \\
\text { cognitivos de la representación de la recompensa, la agresión, la } \\
\text { ansiedad, la depresión, la psicosis, las adicciones y el trastorno } \\
\text { obsesivo-compulsivo } \\
\text { - Participa en la regulación del estado de ánimo negativo }\end{array}$ \\
\hline Oxitocina & $\begin{array}{l}\text { - Es una neurohormona } \\
\text { - Reduce la percepción del dolor y de miedo y aumenta la conexión } \\
\text { emocional con otros } \\
\text { - Favorece la formación de lazos sociales a largo plazo, y por ello } \\
\text { tiene un importante papel en la cognición social }\end{array}$ \\
\hline $\begin{array}{l}\text { Factor de liberación de } \\
\text { Corticotropina (CRF) }\end{array}$ & $\begin{array}{l}\text { - Actúa como neurotransmisor o neuromodulador } \\
\text { - Está distribuida en todo el cerebro, pero principalmente en altas } \\
\text { concentraciones en el núcleo para-ventricular del hipotálamo, el } \\
\text { cerebro anterior basal del complejo amigdalino y el tronco } \\
\text { cerebral. } \\
\text { - Juega un papel importante en las respuestas al estrés, en la } \\
\text { regulación emocional y funciona como analgésico } \\
\text { - Cuando hay una alteración en su liberación se asocia con trastornos } \\
\text { como la ansiedad, depresión, abuso de sustancias psicoactivas y } \\
\text { alcohol }\end{array}$ \\
\hline
\end{tabular}

Fuente. Elaboración propia. 


\subsection{LÍNEAS INVESTIGATIVAS EN NEUROCIENCIAS APLICADAS A LA EDUCACIÓNY ENSEÑANZA- APRENDIZAJE}

Con el avance de las neurociencias, impulsado por la declaración de la década del cerebro, los resultados de las investigaciones en este campo se han venido aplicando a la comprensión de fenómenos y problemas, planteados en diversos campos de conocimiento, como la economía, el derecho, el marketing, y por supuesto la educación. A este último escenario contribuyó la iniciativa "Ciencias del Aprendizaje e Investigación sobre el cerebro" promovida desde 1999 por la Organización para la cooperación y el desarrollo económico (OECD, 2007).

El vínculo entre educación y neurociencias se ha determinado con diversas expresiones, neuroeducación o neurociencias de la educación. Las cuestiones iniciales han trazado su orientación en el estudio y la investigación de las bases neuronales de la lectura, de las habilidades matemáticas y el aprendizaje, así como por el diseño de programas basados en las neurociencias orientados a mejor el rendimiento y las habilidades académicas a través del entrenamiento de funciones como la atención, la memoria, el lenguaje, y las funciones ejecutivas en general (Ansari, De Smedt y Grabner, 2012). El interés ha continuado por el papel que desempeñan las emociones en la formación de competencias socio-emocionales, en el aprendizaje a largo plazo y en el aprendizaje significativo. Esto se ha logrados gracias al uso de técnicas de neuroimagen no invasivas, como la Resonancia Magnética Funcional y Estructural, el electroencefalógrafo (EEG) y el Magnetoencefalógrafo.

La relación entre neurociencias, enseñanza y aprendizaje se ha centrado principalmente en tres líneas: a) el influjo del estrés sobre el aprendizaje (LeDoux, 1996; Pekrun, 2006); b) el papel de las emociones como predictores de los resultados académicos de los estudiantes y de su éxito en la vida laboral y profesional (Ainley, 2006; Artino, La Rochelle y Durning, 2010); c) la importancia del aprendizaje de tareas novedosas.

Sobre la primera línea, Pekrun y Linnenbrink-Garcia (2014) afirman que los entornos educativos generan diversas experiencias afectivas, que existe una relación bidireccional entre el afecto y la cognición, y que las emociones surgidas en los escenarios académicos son resultado de la evaluación del éxito y el fracaso que emergen en estos contextos, así como de las experiencias personales y sociales placenteras o desagradables en encuentros educativos. Estas ideas llevan a Pekrun a proponer la teoría del control-valor de las emociones de logro y sus implicaciones para la investigación y la práctica educativa (Pekrun, 2006).

A través de esta teoría se pretende comprender el efecto de las emociones sobre el rendimiento y el entorno académico. Supone que existen emociones vinculadas a las actividades de logro y a los resultados de logro que median el aprendizaje. Estas emociones tienen el potencial de activar sensaciones de bienestar o malestar, generando el disfrute, frustración, aburrimiento, alegría, esperanza, orgullo, ansiedad, desesperanza, vergüenza e ira relacionados con el éxito o el fracaso (Pekrun, 2006).

Inicialmente esta teoría se estudió con relación a la forma en que los estudiantes enfrentaban eventos estresantes y a la ansiedad que producían ciertas situaciones y actividades académicas (Pekrun, Götz y Perry, 2005). Posteriormente, Pekrun (2006) propone que esta teoría se aplique a la comprensión de las emociones de los estudiantes frente a diferentes actividades académicas: emociones experimentadas por los docentes durante sus prácticas de enseñanza; cooperación profesional; desarrollo de la personalidad; salud psicológica; regulación emocional (Pekrun \& Stephens, 2009), de manera que 
se pueda impactar la institución académica y el bienestar de los estudiantes, docentes, directivos y administrativos, que forman parte de la comunidad académica.

Wolfe (2006) advierte que las actividades realizadas en el aula deben contemplar la posibilidad de que liberen adrenalina en lugar de cortisol, con el fin de atraer el interés emocional y motivacional de los estudiantes. La liberación de adrenalina permite que la experiencia de aprendizaje se almacene en las memorias vívidas, de manera que permanezcan ancladas a la memoria a largo plazo de manera positiva, ya que, como este autor lo señala, cuanto más intensa es la excitación, más fuerte es la huella de memoria. Si se admite que el sistema de memoria tiene un almacén para los hechos comunes y otro para los emocionalmente cargados, este sistema suele olvidar los hechos comunes y mantener a largo plazo los marcados emocionalmente. Por esta razón es importante considerar incluir un ancla emocional al aprendizaje, a través de uso de estrategias como las simulaciones y los juegos de rol.

En la segunda línea se encuentran investigaciones como las de Pekrun (2006), Marchand y Gutiérrez (2012), y Artino, La Rochelle y Durning (2010). Estos investigadores han encontrado que las emociones positivas (disfrute, esperanza y orgullo, entre otras), se asocian con la motivación intrínseca, el esfuerzo, la autorregulación y estrategias de aprendizaje más sofisticadas. Artino et al. (2010) y Marchand y Gutiérrez (2012) han identificado que las emociones negativas (ira, frustración, vergüenza, aburrimiento) se asocian con un esfuerzo reducido, un menor rendimiento, una mayor necesidad de regulación externa y menos estrategias de aprendizaje autorreguladas.

En esta línea, en 1994, el Grupo Fetzer, formado por educadores, pedagogos e investigadores en temas de infancia, introduce el término "Aprendizaje Social y Emocional" (Social and Emotional Learning, SEL), con el fin de promover la formación de competencias sociales y emocionales en la infancia y la adolescencia, esperando que tuvieran un impacto efectivo sobre el rendimiento académico, la salud mental, el bienestar psicológico, la calidad de vida, la capacidades ciudadanas y el comportamiento cívico (Weissberg et al., 2015). Este grupo creo una organización conocida por el nombre de Collaborative for Academic, Social and Emotional Learning (CASEL), cuyo objetivo ha sido aportar prácticas basadas en evidencia sobre el SEL y servir de asesor para priorizar y promover el desarrollo y fortalecimiento de competencias sociales y emocionales desde el preescolar hasta la educación superior, a través de prácticas y políticas educativas (Dusenbury et al., 2014).

El aprendizaje social y emocional (SEL) involucra los procesos a través de los cuales niños y adultos adquieren y aplican efectivamente los conocimientos, actitudes y habilidades necesarias para comprender y manejar las emociones, establecer y alcanzar metas positivas, sentir y mostrar empatía por los demás, establecer y mantener relaciones, y tomar decisiones responsables (National Education Association, 2017; Vassilopoulos, Brouzos y Koutsianou, 2018).

Los programas SEL no sólo incluyen a los estudiantes, sino también a la comunidad académica en general, a las familias y a la comunidad local. Estos programas buscan establecer escenarios académicos positivos, diseñando ambientes seguros de cuidado, cooperación, bienestar y participación (Zins et al., 2004). A través de los programas basados en SEL se potencializan las capacidades de los estudiantes porque integran aspectos cognitivos, emocionales y comportamentales para fortalecer o desarrollar las habilidades, capacidades, competencias, conocimientos y actitudes, que les permiten a las personas hacer frente de manera efectiva a los desafíos y tareas diarias. La aproximación propuesta por CASEL incluye la conciencia de sí mismo, el autoconocimiento, la autogestión, la conciencia social, las habilidades sociales y la toma de decisiones responsable (Weissberg et al., 2015). 
En esta misma línea, la profesora Alison Piepmeier usó la estrategia de incidente crítico para crear un espacio a través del que los estudiantes pudieran expresar sus emociones frente a situaciones que hubieran experimentado y que hubieran generado en ellos fuertes reacciones emocionales. Esta estrategia no sólo se ha usado para que los estudiantes aprendan a gestionar sus emociones, sino que ha servido como estrategia para reflexionar acerca de las formas en las que se pueden enfrentar situaciones críticas de otros, especialmente en aquellos estudiantes que a lo largo de sus prácticas profesionales deben enfrentarse a situaciones de violencia intrafamiliar, guerra, mutilaciones, por mencionar algunas (Eyler, Giles y Schmiede, 1996).

En la tercera línea se ha destacado la importancia de las tareas novedosas que generen retos cognitivos, sobre los procesos de aprendizaje. Se ha señalado que los seres humanos procesan alrededor de cuatrocientos mil millones de bits por segundo, distribuidos en las diferentes tareas que deben ejecutar permanentemente. Esto representa una gran cantidad de información, la mayoría de la cual, no son conscientes de estar procesando. La información procesada, sobre la cual se toma consciencia, es posible sólo si está asociada con un aprendizaje significativo (Wolfe, 2006), desde el cual quien lo haya aprendido tenga la posibilidad de convertirse en un aprendizaje que pueda ser utilizado. Es decir que debe ser un aprendizaje explícito, que plantee retos y esto sólo es posible cuando los procesos de enseñanza aprendizaje involucran la activación de emociones que generen bienestar y planteen retos.

Previamente, Raichle et al. (1994) realizaron un experimento donde demostraban que la práctica de una tarea nueva conduce a un mejor rendimiento. Utilizando una Tomografía por Emisión de Positrones (PET) durante la realización de una tarea novedosa y una repetitiva de selección de respuesta verbal simple (el participante debía decir un verbo apropiado para un sustantivo presentado visualmente), se encontró que la corteza Cingulada anterior (CCA), la corteza prefrontal izquierda, el lóbulo temporal posterior izquierda, y el hemisferio cerebeloso derecho, presentaban una mayor actividad durante la ejecución de la tarea novedosa, en comparación con las tareas de repetición. Los resultados de este estudio mostraron la importancia de las tareas novedosas para el aprendizaje.

Otro estudio centrado en las diferentes actividades cerebrales que se producen ante la presentación de tareas rutinarias o novedosas fue realizado por Gold y Balster (1996). Estos autores demostraron que cuando a las personas se les presentaban estímulos novedosos se activaba el lóbulo frontal derecho, y cuando esta tarea se volvía rutinaria, sólo se veía actividad en el lóbulo frontal izquierdo. Esta actividad del lóbulo frontal derecho se asociaba con una mayor capacidad de atención sostenida por períodos más largos. En la medida en que las personas se familiarizan con la tarea, el lóbulo frontal izquierdo toma el control y disminuyen los tiempos de atención. Frente a los procesos de enseñanza aprendizaje, esto indica que los estudiantes deben involucrarse continuamente en tareas novedosas que los motive a aprender y que los mantenga a la expectativa frente al aprendizaje de nuevos conocimientos. Cuando un profesor no genera expectativa es más fácil que aparezca el aburrimiento y la desmotivación.

\section{DISCUSIÓN}

Conocer las estructuras cerebrales y los neurotransmisores que participan en los procesos que vinculan cognición y emoción, permite a las personas responsables de los diseños curriculares y de la evaluación de los procesos de enseñanza-aprendizaje, pensar, diseñar 
e implementar actividades académicas basadas en los hallazgos de las neurociencias, de forma tal que, como lo ha evidenciado Pekrun en sus distintas investigaciones, no se generen situaciones que incrementen los niveles de distrés que conduzcan a la deserción académica en la vida universitaria.

Estos hallazgos presentan retos para los neurocientíficos interesados en las investigaciones sobre la importancia de las emociones en los procesos de enseñanza aprendizaje, en tanto son pocas las investigaciones experimentales con técnicas de neuroimagen a nivel de la educación superior que expliquen cómo los procesos de enseñanza aprendizaje están favoreciendo la permanencia académica y el éxito profesional. Asimismo, el desafío de no obviar los ambientes y escenarios educativos en las mencionadas investigaciones experimentales, lo que permitiría una mayor efectividad en sus resultados al considerar las diversas dimensiones de los procesos emocionales.

Por otra parte, sería necesario comenzar por dar un giro hacia los escenarios universitarios para promover el desarrollo y fortalecimiento de competencias emocionales y sociales en estudiantes de educación superior, mediante el currículo como elemento trasversal (Núñez, 2006; Álvarez et al., 2000); los syllabus de las asignaturas que reflejen las competencias generales y especificas (Álvarez et al., 2000); las tutorías académicas, administrativas, psicosociales y profesionales desde donde se brinda apoyo a los estudiantes y se acompañan los procesos de formación (Bisquerra, 2001; Bisquerra y Pérez, 2007; Bisquerra, 2006); los espacios académicos electivos que flexibilizan el currículo y permiten la inclusión de contenidos académicos trasversales (Ben-Shahar, 2006; Álvarez et al., 2000; Bisquerra y Pérez, 2007); las prácticas profesionales en las que se puede acompañar y fortalecer competencias más allá de las cognitivas; los espacios informales donde se dan interacciones entre estudiantes, profesores y administrativos, como los pasillos de las universidades, las cafeterías, los cine- foros, las presentaciones artísticas, los eventos culturales, entre otros (Felten et al., 2006).

Asimismo, es importante imaginar los diferentes escenarios universitarios, como escenarios sociales, donde el aprendizaje no sólo se da gracias a las diferencias individuales, sino también como producto de las interacciones que se producen entre las personas que integran estos ambientes, en los cuales no sólo es relevante la formación de competencias académicas, sino de competencias y habilidades socioemocionales que favorezcan un mejor desempeño y compromiso con el ejercicio profesional.

Por otra parte, es importante el desarrollo de un mayor número de investigaciones empíricas que se apoyen en el uso de las herramientas y tecnologías que puede aportar las neurociencias, así como de revisiones sistemáticas y metaanálisis, que permitan identificar las mejores estrategias para dar cumplimiento a los objetivos pretendidos a través de la formación universitaria. Estas contribuciones también se podrían orientar a diseños de políticas, planes y programas en la educación superior, que redunde en una transformación de la sociedad en general, en tanto se puede garantizar la formación de profesionales con competencias cognitivas, afectivas y sociales capaces de trasformar su realidad.

\section{CONCLUSIONES}

Las investigaciones basadas en las diferentes teorías han permitido identificar las bases neuroemocionales del aprendizaje y la enseñanza. Por ahora, se sabe que los procesos 
neurocognitivos y neuroemocionales que subyacen al aprendizaje son: la sensación y la percepción, los mecanismos atencionales, el pensamiento contrafáctico, el procesamiento de la información, los mecanismos neuropsicológicos de la memoria, el proceso de toma de decisiones, el procesamiento automático versus el procesamiento controlado, los mecanismos del aprendizaje colaborativo, implícito, explicito, el sistema de recompras y el procesamiento de información novedosa versus el procesamiento de información rutinaria (Flagel et al., 2011; Knudsen, 2004).

En este artículo se exploraron aplicaciones, desde el campo de las neurociencias sociales y afectivas, a la formación de competencias socioemocionales en escenarios universitarios, así como las relaciones entre las emociones y los procesos de enseñanza aprendizaje. Entre los temas examinados se incluyeron la integración, el bajo rendimiento, el autoconcepto académico, los programas de cambio de logro, las emociones relacionadas con el logro, la alabanza y culpa, la ayuda y las comunicaciones afectivas de compasión y enojo. En el desarrollo de la revisión investigativa se reconoce, que las neurociencias por sí solas no tienen las explicaciones para todos los fenómenos, pero está en capacidad de proporcionar información sobre las habilidades y las limitaciones del cerebro frente al aprendizaje.

La revisión permitió determinar un alto número de publicaciones sobre la importancia de incluir las emociones en el estudio de las practicas pedagógicas, sin embargo, hasta la fecha existe poca evidencia empírica neurocientífica sobre el efecto de estrategias pedagógicas que involucran el aspecto emocional sobre la adaptación y permanencia de los estudiantes a la vida universitaria y el desarrollo de las competencias generales y específicas que deban adquirir los futuros profesionales. La mayoría de las publicaciones son teóricas o de reflexión, y se han centrado sobre prácticas pedagógicas en preescolar, primaria y bachillerato, o en estudios con profesores. No obstante, los avances en las investigaciones en este campo, particularmente en situaciones de laboratorio o en pacientes con daño cerebral, nos conducen a un cambio en la redefinición de las prácticas pedagógicas en el ámbito universitario (Felten, Gilchrist y Darby, 2006).

En contraste con la importancia de los hallazgos evidenciados en las investigaciones revisadas, son pocas las investigaciones experimentales con validez ecológica que se han desarrollado. Los descubrimientos y aportes de las neurociencias sobre las emociones ofrecen explicaciones significativas que resultan relevantes para la teoría y la práctica educativa, no obstante, sin investigaciones en contextos reales, en ambientes educativos y de aula, no podría revisarse la efectividad de muchas de las prácticas pedagógicas, ni tener un mayor conocimiento sobre las condiciones bajo las cuales, los estudiantes de educación superior, aprenden y desarrollan competencias que les permitan tener éxito en su vida laboral. Es por esto que el reto más cercano y el horizonte que permanece abierto son propuestas para desarrollar investigaciones ecológicamente válidas sobre las prácticas pedagógicas en educación superior, desde las neurociencias, sobre la relación entre cognición y emoción.

\section{REFERENCIAS BIBLIOGRÁFICAS}

Adolphs R. (2017). How should neuroscience study emotions? by distinguishing emotion states, concepts, and experiences. Social Cognitive and Affective Neuroscience, 12(1), 24-31.

Ainley, M. (2006). Connecting with learning: Motivation, affect and cognition in interest processes. Educational Psychology Review, 18(4), 391-405. 
Álvarez, M., Bisquerra, R., Fita, E., Martínez, F., y Pérez, N. (2000). Evaluación de programas de educación emocional. Revista de investigación educativa, 18(2), 587-599.

Ansari, D., De Smedt, B., \& Grabner, R. H. (2012). Neuroeducation -a critical overview of an emerging field. Neuroethics, 5(2), 105-117.

Ariza, G. I., y Ocampo, H. B. (2005). El acompañamiento tutorial como estrategia de la formación personal y profesional: un estudio basado en la experiencia en una institución de educación superior. Universitas Psychologica, 4(1), 31-42.

Arroyo, R. (2012). Habilidades gerenciales: desarrollo de destrezas, competencias y actitud. Bogotá: ECOE.

Artino, A. R., La Rochelle, J. S., \& Durning, S. J. (2010). Second-year medical students' motivational beliefs, emotions, and achievement. Medical Education, 44(12), 1203-1212.

Bar-On, R. E., \& Parker, J. D. (Eds.). (2000). The Handbook of Emotional Intelligence: Theory, Development, Assessment, and Application at Home, School, and in the Workplace. Jossey-Bass.

Beneitone, P., Esquetini, C., González, J., Marty, M., Siufi, G. y Wagenaar, R. (2007). Reflexiones y perspectivas de la educación superior en América Latina: informe final Proyecto Tuning América Latina: 2004-2007. Universidad de Deusto / Universidad de Groningen.

Ben-Shahar, T. (2006). Positive Psychology, 1504. Última visita 30 noviembre de 2018. Recuperado desde https://positivepsychologyprogram.com/wp-content/uploads/2013/12/positive-psychology1504-syllabus.pdf.

Bisquerra, R. (2006). Orientación psicopedagógica y educación emocional. Estudio sobre educación, (11), 9-25.

. (2001). Orientación psicopedagógica y educación emocional en la educación formal y no formal. Ágora digital, (2). Última visita 9 de enero de 2019. Recuperado desde http://rabida.uhu. es/dspace/bitstream/handle/10272/3448/b15760364.pdf?sequence $=1$

Bisquerra, R. y Pérez, N. (2007). Las competencias emocionales. Educación XX1, vol.10, 61-82.

Cacioppo, J. T., \& Cacioppo, S. (2013). Social neuroscience. Perspectives on Psychological Science, $8(6), 667-669$.

Cacioppo, J. T., Norris, C. J., Decety, J., Monteleone, G. \& Nusbaum, H. (2009). In the eye of the beholder: individual differences in perceived social isolation predict regional brain activation to social stimuli. Journal of Cognitive Neuroscience, 21(1), 83-92.

Carew, T. J., \& Magsamen, S. H. (2010). Neuroscience and education: An ideal partnership for producing evidence-based solutions to guide 21st century learning. Neuron, 67(5), 685-688.

Cerezo, A., Ceca, M. J. B., \& Blanco, J. L. Y. (2018). Traceability of Intra-and Interpersonal Skills: From Education to Labor Market. In Otero-Mateo, M. \& Pastor-Fernández, A. Human Capital and Competences in Project Management (pp. 87-110). InTechOpen. Última visita 12 de diciembre de 2018. Recuperado desde https://cdn.intechopen.com/pdfs/57566.pdf

Chernyshenko, O., Kankaraš, M. \& Drasgow, F. (2018). Social and emotional skills for student success and wellbeing: Conceptual framework for the OECD study on social and emotional skills. OECD Education Working Papers, No. 173, OECD Publishing, Paris.

Chóliz, M. (2005). Psicología de la emoción: el proceso emocional. Dpto de Psicología Básica. Universidad de Valencia. Última visita 13 de diciembre de 2018. Recuperado desde https://www. uv.es/choliz/Proceso\%20emocional.pdf

Damasio, A. (2005). Human behaviour: brain trust. Nature, 435(7042), 571-572. . (1999). The feeling of what happens: Body and emotion in the making of consciousness. New York: Harcourt Brace.

(1994). El error de Descartes: la razón de las emociones. Andrés Bello.

Damasio, H., Grabowski, T. J., Tranel, D., Hichwa, R. D. \& Damasio, A. R. (1996). A neural basis for lexical retrieval. Nature, 380(6574), 499-505.

Deak, A. (2011). Brain and emotion: Cognitive neuroscience of emotions. Review of Psychology, 18(2), 71-80. 
Decety, J. E. \& Ickes, W. E. (eds.) (2009). The social neuroscience of empathy. MIT Press.

Dusenbury, L., Weissberg, R. P., Goren, P. \& Domitrovich, C. (2014). State Standards to Advance Social and Emotional Learning: Findings from CASEL's State Scan of Social and Emotional Learning Standards, Preschool through High School, 2014. Collaborative for Academic, Social, and Emotional Learning. Última visita 9 de enero de 2019. Recuperado desde: https://casel.org/ wp-content/uploads/2016/06/casel-brief-on-state-standards-january-2014.pdf

Eyler, J., Giles, D. E. Jr. \& Schmiede, A. (1996). A practitioner's guide to reflection in service-learning: Student voices \& reflections. Nashville: Vanderbilt University. Última visita 9 de enero de 2019. Recuperado desde: https://leduccenter.files.wordpress.com/2015/02/practitioners-guide-toreflection-in-service-learning.pdf

Felten, P., Gilchrist, L. Z. \& Darby, A. (2006). Emotion and Learning: Feeling Our Way toward a New Theory of Reflection in Service-Learning. Journal of Community Service Learning, 12(2), 38-46.

Flagel, S. B., Clark, J. J., Robinson, T. E., Mayo, L., Czuj, A., Willuhn, I., Akers, Ch. A., Clinton, S. M., Phillips, P. E. \& Akil, H. (2011). A selective role for dopamine in stimulus-reward learning. Nature, 469(7328), 53-57.

Fuster, J. M. (2003). Cortex and mind: Unifying cognition. Oxford: University Press.

Gallart, M. (1997). Los cambios en la relación escuela-mundo laboral. Revista Iberoamericana de educación, 15, 159-174.

Gazzaniga, M. S. (2004). The cognitive neurosciences. MIT Press.

George, J. M., \& Dane, E. (2016). Affect, emotion, and decision making. Organizational Behavior and Human Decision Processes, 136, 47-55.

Gold, L. H. \& Balster, R. L. (1996). Evaluation of the cocaine-like discriminative stimulus effects and reinforcing effects of modafinil. Psychopharmacology, 126(4), 286-292.

Gray, J. A. (1990). Brain systems that mediate both emotion and cognition. Cognition and Emotion, 4(3), 269-288.

Gutiérrez, N. B., Zalba, E. M. y Deamini, C. (2010). Educación basada en competencias: desarrollos conceptuales y experiencias en la Universidad Nacional de Cuyo y otros enfoques. Mendoza: EDIUNC.

Han, H., Chen, J., Jeong, C. \& Glover, G. H. (2016). Influence of the cortical midline structures on moral emotion and motivation in moral decision-making. Behavioural Brain Research, 302, 237-251.

Howard-Jones, P. A. (2011). A multiperspective approach to neuroeducational research. Educational Philosophy and Theory, 43(1), 24-30.

Ibanez, A., Kotz, S. A., Barrett, L., Moll, J. \& Ruz, M. (2014). Situated affective and social neuroscience. Frontiers in human neuroscience, 8, Article 547. Última visita 9 de enero de 2019. Recuperado desde https://www.frontiersin.org/articles/10.3389/fnhum.2014.00547/full

Immordino-Yang, M. H. (2011). Implications of affective and social neuroscience for educational theory. Educational Philosophy and Theory, 43(1), 98-103.

Immordino-Yang, M. H. \& Damasio, A. (2007). We feel, therefore we learn: The relevance of affective and social neuroscience to education. Mind, Brain, and Education, 1(1), 3-10.

Izard, C. E. (1979). The maximally discriminative facial movement coding system (Max). Newark: University of Delaware.

. (1971). The face of emotion. New York: Appleton-Century-Crofts.

James, W. (1884). What is an emotion? Mind, 9, 188-205.

Jankowski, K. F. \& Takahashi, H. (2014). Cognitive neuroscience of social emotions and implications for psychopathology: examining embarrassment, guilt, envy, and schadenfreude. Psychiatry and Clinical Neurosciences, 68(5), 319-336.

Knudsen, E. I. (2004). Sensitive periods in the development of the brain and behavior. Journal of Cognitive Neuroscience, 16(8), 1412-1425.

Lang, P. J. (1995). The emotion probe: studies of motivation and attention. American Psychologist, 50(5), 372-385. 
Lasnier, F. (2000). Réussir la formation par compétences. Montreal: Guérin.

Lazarus, R. S. (1982). Thoughts on the relations between emotion and cognition. American Psychologist, 37(9), 1019-1024.

Le Boterf, G. (2001). L'ingénierie des competénces. Paris: Éditions d'organisation.

LeDoux, J. E. (2012). Rethinking the emotional brain. Neuron, 73(4), 653-676. (1996). The emotional brain. New York: Simon \& Schuster.

(1989). Cognitive-emotional interactions in the brain. Cognition \& Emotion, 3(4), 267-289.

LeDoux, J. E. \& Phelps, E. A. (2000). Emotional networks in the brain. In: Lewis M, Haviland-Jones JM (eds.). Handbook of Emotions (pp. 157-172). New York: Guilford.

LeDoux, J. E., Wilson, D. H., \& Gazzaniga, M. S. (1978). Block Design performance following callosal sectioning: observations on functional recovery. Archives of Neurology, 35(8), 506-508.

Lévy-Leboyer, C. (2000). Gestión de las competencias: cómo analizarlas, cómo evaluarlas y cómo desarrollarlas. Barcelona: Gestión.

Lindsley, D. B. (1951). Emotion. In S. S. Stevens (Ed.). Handbook of Experimental Psychology (pp. 473-516). Oxford: Wiley.

MacLean, P. D. (1990). The triune brain in evolution: Role in paleocerebral functions. US: Springer Science \& Business Media.

Marchand, G. C. \& Gutierrez, A. P. (2012). The role of emotion in the learning process: Comparisons between online and face-to-face learning settings. The Internet and Higher Education, 15(3), 150-160.

Meltzoff, A. N., Kuhl, P. K., Movellan, J. \& Sejnowski, T. J. (2009). Foundations for a new science of learning. Science, 325(5938), 284-288.

Moll, J., De Oliveira-Souza, R. \& Eslinger, P. J. (2003). Morals and the human brain: a working model. Neuroreport, 14(3), 299-305.

National Education Association (2017). The Importance of Social Emotional Learning for All Students Across All Grades. Última visita 9 de enero de 2019. Recuperado desde https://files. eric.ed.gov/fulltext/ED581059.pdf

Núñez, L. (2006). Pedagogía emocional: una experiencia de formación en competencias emocionales en el contexto universitario. Cuestiones Pedagógicas, 18, 65-80.

OECD (2015). Skills for Social Progress: The Power of Social and Emotional Skills. OECD Skills Studies.

OECD. (2007). Understanding the brain: The birth of a learning science. Última visita 9 de enero de 2019. Recuperado desde: https://www.oecd.org/site/educeri21st/40554190.pdf

Panksepp, J. (2003). At the interface of the affective, behavioral, and cognitive neurosciences: Decoding the emotional feelings of the brain. Brain and Cognition, 52(1), 4-14.

Panksepp, J. \& Biven, L. (2012). The archaeology of mind: Neuroevolutionary origins of human emotions. New York: WW Norton \& Company.

Paoloni, P. (2014). Emociones en contextos académicos: perspectivas teóricas e implicaciones para la práctica educativa en la Universidad. Universidad de Almería. Electronic Journal of Research in Educational Psychology, 12(3), 567-596. Última visita 9 de enero de 2019. Recuperado desde http://ri.conicet.gov.ar/bitstream/handle/11336/35216/CONICET_Digital_Nro.9cf57c27-a0924616-bcb8-bac44d582197_A.pdf?sequence=2\&isAllowed=y

Prada, M. D. \& González, J. (2014). Competence-based multiple learning paths: on the road of implementation. Tuning Journal for Higher Education, 2(1), 107-128.

Pekrun, R. (2006). The control-value theory of achievement emotions: Assumptions, corollaries, and implications for educational research and practice. Educational Psychology Review, 18(4), 315-341.

Pekrun, R., Götz, T. \& Perry, R. P. (2005). Achievement emotions questionnaire (AEQ). User's manual. Unpublished Manuscript, University of Munich, Munich

Pekrun, R. \& Linnenbrink-Garcia, L. (2014). Introduction to emotions in education. In Pekrun, R., \& Linnenbrink-Garcia, L. (eds.). International Handbook of Emotions in Education (pp. 11-20). Routledge. 
Pekrun, R. \& Stephens, E. (2009). Goals, Emotions, and Emotion Regulation: Perspectives of the Control-Value Theory. Human Development, 52(6), 357- 365.

Pessoa L. (2017). A Network Model of the Emotional Brain. Trends in cognitive sciences, 21(5), 357-371.

Pletti, C., Lotto, L., Buodo, G. \& Sarlo, M. (2017). It's immoral, but I'd do it! Psychopathy traits affect decision-making in sacrificial dilemmas and in everyday moral situations. British Journal of Psychology, 108(2), 351-368.

Plutchik, R. (1980). A general psychoevolutionary theory of emotion. In Plutchik, R. \& Kellerman, H. (Eds.). Emotion: Theory, research, and experience: Vol. 1. Theories of Emotion (pp. 3-33). New York: Academic.

Raichle, M. E., Fiez, J. A., Videen, T. O., MacLeod, A. M. K., Pardo, J. V., Fox, P. T. \& Petersen, S. E. (1994). Practice-related changes in human brain functional anatomy during nonmotor learning. Cerebral Cortex, 4(1), 8-26.

Rolls, E. T. (2000). On the brain and emotion. Behavioral and brain sciences, 23(2), 219-228.

Rychen, D. S. y Salganik, L. H. (2006). Las competencias clave para el bienestar personal, social y económico. Málaga: Aljibe.

Schachter, S. \& Singer, J. (1962). Cognitive, social, and physiological determinants of emotional state. Psychological Review, 69(5), 379-399.

Taylor, R. D., Oberle, E., Durlak, J. A. \& Weissberg, R. P. (2017). Promoting positive youth development through school-based social and emotional learning interventions: A meta-analysis of follow-up effects. Child Development, 88(4), 1156-1171.

Torres, A., Catena, A., Megías, A., Maldonado, A., Cándido, A., Verdejo-García, A. \& Perales, J. C. (2013). Emotional and non-emotional pathways to impulsive behavior and addiction. Frontiers in Human Neuroscience, 7, Article ID 43. Última visita 9 de enero de 2019. Recuperado desde http://psycnet.apa.org/record/2013-15799-001

Uchino, B. N., Cacioppo, J. T. \& Kiecolt-Glaser, J. K. (1996). The relationship between social support and physiological processes: a review with emphasis on underlying mechanisms and implications for health. Psychological Bulletin, 119(3), 488-531.

Vassilopoulos, S. P., Brouzos, A. \& Koutsianou, A. (2018). Outcomes of a universal social and emotional learning (SEL) group for facilitating first-grade students' school adjustment. International Journal of School \& Educational Psychology, 6(3), 223-236.

Vohs, K. D., Baumeister, R. F., Schmeichel, B. J., Twenge, J. M., Nelson, N. M. \& Tice, D. M. (2008). Making choices impairs subsequent self-control: A limited-resource account of decision making, self-regulation, and active initiative. Journal of Personality and Social Psychology, 94(5), 883-898.

Vuilleumier, P. (2005). How brains beware: neural mechanisms of emotional attention. Trends in Cognitive Sciences, 9(12), 585-594.

WEF, World Economic Forum (2016). New Vision for Education: Fostering Social and Emotional Learning through Technology. Informe. Última visita 14 de diciembre de 2018. Recuperado desde http://www3.weforum.org/docs/WEF_New_Vision_for_Education.pdf

Weissberg, R. P., Durlak, J. A., Domitrovich, C. E. \& Gullotta, T. P. (2015). Social and emotional learning: Past, present, and future. In: Durlak, J. A., Domitrovich, C. E., Weissberg, R. P. \& Gullotta, T. P. (Eds.). Handbook of social and emotional learning: Research and practice (pp. 3-19). New York: Guilford Press.

Wolfe, P. (2006). The role of meaning and emotion in learning. New directions for adult and continuing education, 110, 35-41.

Zajonc, R. B. (1980). Feeling and thinking: Preferences need no inferences. American Psychologist, 35(2), 151-175.

Zins, J. E., Weissberg, R. P., Wang, M. C. \& Walberg, H. J. (eds.). (2004). Building academic success on social and emotional learning: What does the research say? New York: Teachers College Press. 